\title{
Filtering Mechanism for Unwanted Messages on OSN User Wall Using CBMF (Content Based Message Filtering)
}

\author{
Aishwarya Kashyup ${ }^{1}$, Himanshu Kothari ${ }^{2}$,Ravina Kothari ${ }^{3}$, Geetika Narang ${ }^{4}$ \\ Last year student B.E., Computer Engineering, SIT, Lonavala, India ${ }^{1,2,3}$ \\ Associate Professor, Computer Engineering, SIT, Lonavala, India ${ }^{4}$
}

\begin{abstract}
In this era of internet, the Online Social Networks (OSNs) are the platform to build social relations among people who share interests, activities, backgrounds or real life connections. OSNs have gained a ubiquitous status and this has led to security issues of posting unwanted messages on user wall. Therefore, in order to make the OSN user wall a secured wall, we are introducing a flexible- rule based system which provides users to control the messages that are posted on their walls and allows user to customise the filtering criteria to be applied on their walls. This system exploits machine learning based soft classifier for automatically labelling messages in support of content based filtering.
\end{abstract}

Keywords: Online Social Networks, Flexible-rule based system, filtering criteria, Machine - learning based soft classifier, content based filtering.

\section{I.INTRODUCTION}

The Online Social networks have become a necessity in Our model implements a hierarchical two level our daily life. The OSNs are a popular medium of classification strategy. In the first level the RBFN interaction where large amounts of human information are classifies the messages into neutral and non-neutral. In the disseminated. Several contents such as audio, video, texts, second level, the non-neutral messages are classified images etc are shared on a daily basis. The statistics of producing gradual estimates of appropriateness to each facebook says, an average user creates 90 pieces of category. After the classification is done, the filtering rules contents each month, whereas more than 30 billion pieces are applied.

of content are shared each month. This has led to the breach of privacy on OSN user wall and as a consequence the user might face the issue of unwanted messages on their walls.

Therefore in order to prevent the unwanted messages to be posted on OSN user wall information filtering is used. Thus, the proposed system uses a flexible rule based filtering that allows user to customise the filtering criteria that is to be applied to their walls.

This service is not only a matter of using web content mining techniques for different application, rather it requires to design additional classification strategies and also wall post comprises of short text and traditional methods have limitations. Here the short texts are separated and categorised based on its content using machine learning text categorization that automatically assigns short texts to a set of categories.

The motive of this work is to propose an automated system called Filtered Wall (FW). This system provides the user the privilege to control the messages that are posted on their walls.

We make use of neural learning as far as learning model is concerned. This is one of the best solutions in text classification. We base the overall short text classification strategy on RBFN for their proven capabilities in acting as soft classifier.

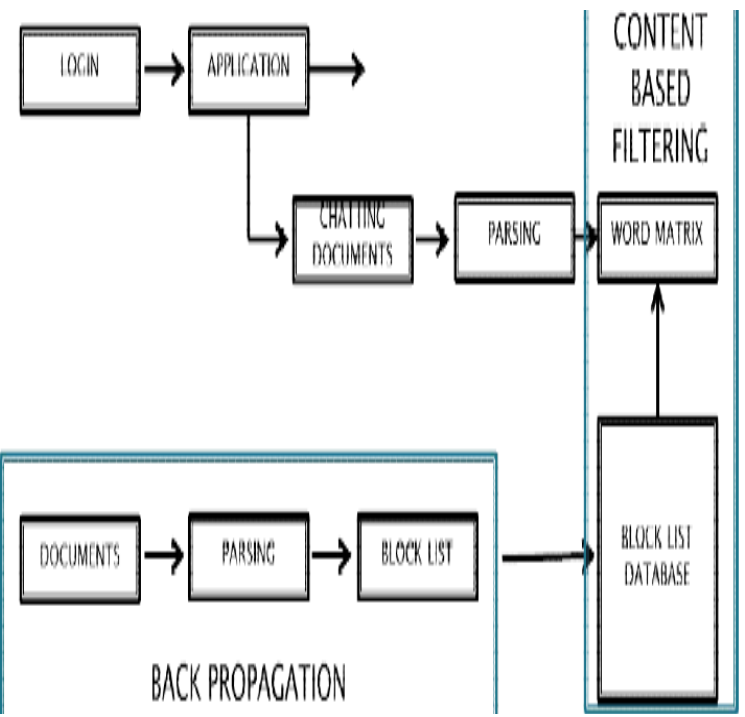

Fig.1 Filtered Wall Architecture

Filtering rules give the result of ML categorisation process, which filter the user wall and relationship of user. In addition, the system provides the support for user temporarily prevented to post any kind of messages on OSN user wall. defined black lists (BLs) that is lists of users that are 


\section{II.SYSTEM ARCHITECTURE}

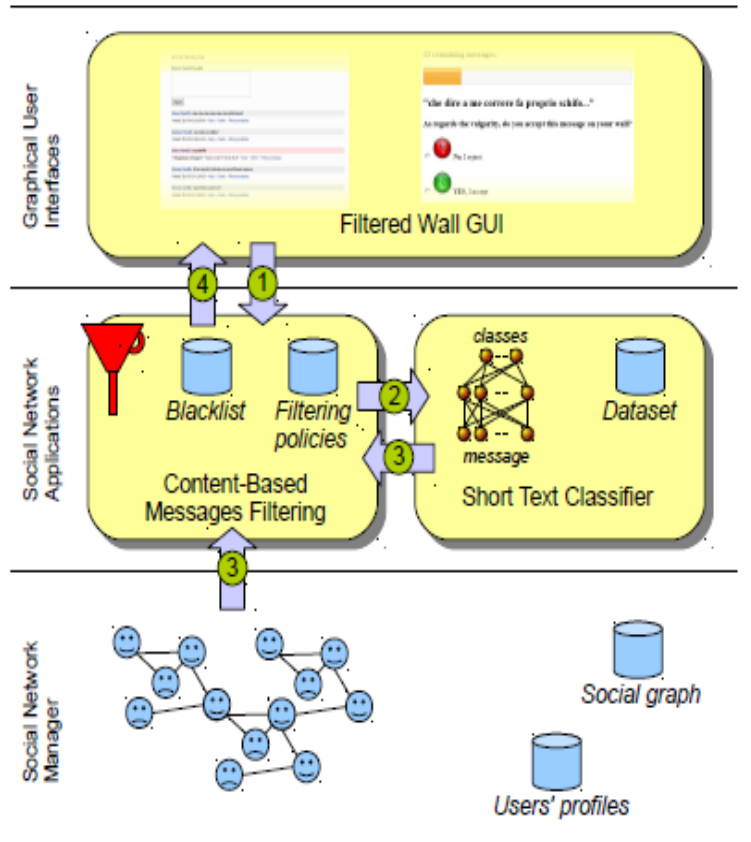

Fig.2. Filtered wall conceptual architecture and the flow messages follow, from writing to publication.

The osn architecture is three-tier architecture. The first layer of the architecture is Social Network Manager (SNM). The aim of SNM is to provide basic OSN functionalities (i.e. profile and relationship management). The second layer provides the support for external Social Network Applications (SNAs). SNAs may in turn require an additional layer for graphical user interface (GUIs). According to the architecture the proposed system is placed in the second and third layers. The users interact through GUIs, which provides users with FW, a wall where messages authorised by FRs/BLs are published. The fundamental components of the proposed system are Content-Based Message Filtering and Short Text Classifier (STC) modules. the latter classifies the messages into different categories. CBMF exploits the messages classified by STC in order to enforce FRs specified by the user. BLs can also be added to improve the filtering process. Given below is the summarised working of the given architecture:

1) After entering the private wall of one of his/her contacts, the user tries to post a message, which is intercepted by $\mathrm{FW}$.

2) A ML-based text classifier extracts metadata from the content of the message.

3) FW uses metadata provided by the classifier, together with data extracted from the social graph and users' profiles, to enforce the filtering and BL rules.

4) Depending on the result of the previous step, the message will be published or filtered by FW.

\section{A. Content-Based Filtering}

Information filtering systems are designed to classify a stream of dynamically generated information dispatched asynchronously by an information producer and present to the user those information that are likely to satisfy his/her requirements. In content-based filtering, each user is assumed to operate independently. As a result, a contentbased filtering system selects information items based on the correlation between the content of the items and the user preferences as opposed to a collaborative filtering system that chooses items based on the correlation between people with similar preferences. While electronic mail was the original domain of early work on information filtering, subsequent papers have addressed diversified domains including newswire articles, Internet "news" articles, and broader network resources. Documents processed in content-based filtering are mostly textual in nature and this makes content-based filtering close to text classification. The activity of filtering can be modelled, in fact, as a case of single label, binary classification, partitioning incoming documents into relevant and non relevant categories. More complex filtering systems include multi-label text categorization automatically labelling messages into partial thematic categories. Content-based filtering is mainly based on the use of the ML paradigm according to which a classifier is automatically induced by learning from a set of preclassified examples. A remarkable variety of related work has recently appeared which differ for the adopted feature extraction methods, model learning, and collection of samples. The feature extraction procedure maps text into a compact representation of its content and is uniformly applied to training and generalization phases. Several experiments prove that Bag-of-Words (BoW) approaches yield good performance and prevail in general over more sophisticated text representation that may have superior semantics but lower statistical quality. As far as the learning model is concerned, there are a number of major approaches in content-based filtering and text classification in general showing mutual advantages and disadvantages in function of application dependent issues. In a detailed comparison analysis has been conducted confirming superiority of Boosting-based classifiers, Neural Networks and Support Vector Machines over other popular methods, such as Rocchio and Naive Bayesia. However, it is worth to note that most of the work related to text filtering by ML has been applied for long-form text and the assessed performance of the text classification methods strictly depends on the nature of textual documents.

\section{B. $\quad$ Short Text Classifier}

Established techniques used for text classification work well on data sets with large documents such as newswires corpora, but suffer when the documents in the corpus are short. In this context, critical aspects are the definition of a set of characterizing and discriminate features allowing the representation of underlying concepts and the collection of a complete and consistent set of supervised examples. Our study is aimed at designing and evaluating various representation techniques in combination with a neural learning strategy to semantically categorize short texts.

From a ML point of view, we approach the task by defining a hierarchical two-level strategy assuming that it is better to identify and eliminate "neutral" sentences, then 
classify "non-neutral" sentences by the class of interest • instead of doing everything in one step. This choice is motivated by related work showing advantages in classifying text and/or short texts using a hierarchical strategy. The first-level task is conceived as a hard classification in which short texts are labelled with crisp Neutral and Non-neutral labels. The second-level soft classifier acts on the crisp set of non-neutral short texts and, for each of them it "simply" produces estimated appropriateness or "gradual membership" for each of the conceived classes, without taking any "hard" decision on any of them. Such a list of grades is then used by the subsequent phases of the filtering process.

\section{ANALYSIS OF THE PROBLEM}

A system will automatically filter unwanted messages from OSN user walls on the basis of both message content and the message creator relationships and characteristics. The paper substantially extends for what concerns both the rule layer and the classification module. Major differences include, a different semantics for filtering rules to better fit the considered domain, an online setup assistant (OSA) to help users in FR specification, the extension of the set of features considered in the classification process, a more deep performance evaluation study and an update of the prototype implementation to reflect the changes made to the classification techniques. In web mining the most general sense it can contribute to the increase of profits, be it by actually selling more products or services, or by minimizing the costs. In order to do this, marketing intelligence is required. This intelligence can focus on marketing strategies and competitive analyses or on the relationship with the customers. The different kinds of web data that are somehow related to customers will then be categorized and clustered to build detailed customer profiles. This not only helps companies to retain current customers by being able to provide more personalized services, but it also contributes in the search for potential customers.

\section{PROPOSED WORK}

- The application is user friendly with various controls provided by the system rich user interface.

- The system makes the overall project management much easier and flexible.

- $\quad$ It can be accessed over the Intranet.

- The system maintains a centralised database which stores user information.

- Therefore, the user information is secured as the data is not stored in the client machine.

- Authentication is provided for this application only registered Users can access.

- There is no risk of data management at any level while the project development is under process.

- The computers are classified under different categories in the database to easily identify various computer peripherals.

- The system gives users the privilege to filter the messages posted on their walls using the filtering rules.
The messages are first classified into different categories and then filtering rules are applied to it.

- BLs are the added features that improve the filtering rules.

\section{V.IMPLEMENTATION}

The proposed system provides the user a feature that automatically filters the unwanted messages on the basis of both message content and the message creator relationships and characteristics. The paper considerably extends for what concerns both the rule layer and the classification module to automatically assign with each short text message a set of categories based on its content.

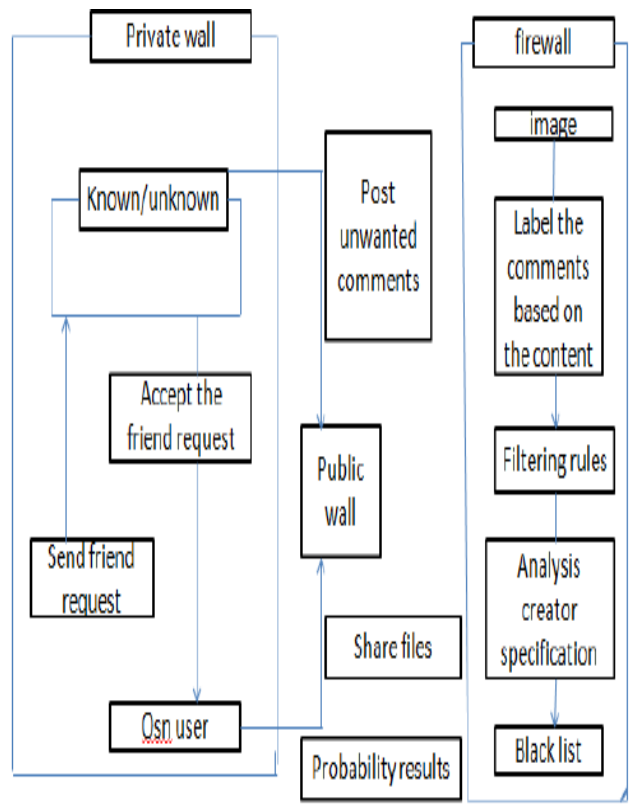

Fig. 2 Architecture diagram

Additionally, we plan to investigate the use of online learning paradigms able to include label feedbacks from users and we plan to enhance our system with more sophisticated to decide when a user should be inserted into a BL. The major efforts in building a robust short text classifier are concentrated in the extraction and selection of a set of characterizing and discriminate features. The solutions investigated in this paper are an extension of those adopted in a previous work by us from which we inherit the learning model and the elicitation procedure for generating pre-classified data. The original set of features, derived from endogenous properties of short texts, is enlarged here including exogenous knowledge related to the context from which the messages originate. As far as the learning model is concerned, we confirm in the current paper the use of neural learning which is today recognized as one of the most efficient solutions in text classification. In particular, we base the overall short text classification strategy on Radial Basis Function Networks (RBFN) for their proven capabilities in acting as soft classifiers, in managing noisy data and intrinsically vague classes. Moreover, the speed in performing the learning phase creates the premise for an adequate use in OSN domains, 
as well as facilitates the experimental evaluation tasks. The goal of the paper is therefore to propose and experimentally evaluate an automated system, called Filtered Wall. System proposed Machine Learning (ML) text categorization techniques. Create neural model within a hierarchical two level classification strategy. Generate user-defined Blacklists (BLs) module.

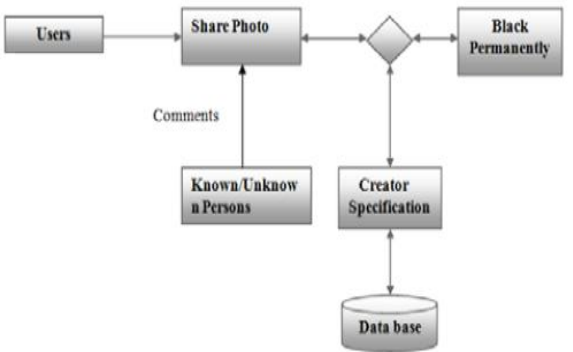

Fig.3 Blacklist System

\section{LITERATURE REVIEW}

Ms. Shruti C. Belsare, Prof. R.R. Keole, "OSN user filtered walls for unwanted messages using content mining"[1], march-2014, proposed a system that will automatically filter unwanted messages from OSN user walls on the basis of both message content and message creator relationships and characteristics. The limitation of this paper is that the users will have no privilege on FR specification.

Dipali D. Vidhate, Ajay P. Thakare,'to avoid unwanted messages from osn user wall: content based filtering approach"[2], april 2014, concerns both the rule layer and the classification module. The limitation in this paper is that the analysis of related work has highlighted the lack of a publicly available benchmark for comparing different approaches to content based classification of user walls short texts.

R. J. Mooney and L. Roy, "Content-based book recommending using learning for text categorization," [3], 2000, uses Collaborative filtering method, but in the proposed system content based recommendation is used. It explains a content based book recommending system that develops information extraction and machine learning algorithm for text categorization.

M. Vanetti, E. Binaghi, B. Carminati, M. Carullo, and E. Ferrari, "Content-based filtering in on-line social networks," [4], 2010, Quality of classification is considered as the main aim. This system can usually take decision about the messages which is blocked, due to the tolerance depends on statistical data.

F. Sebastiani, "Machine learning in automated text categorization," [5], 2002, Efficiency is good, labour power will be saved is the advantage of this paper. The main approach used here is text categorization. Comparison will be performed between human expert and labour power expert.

H. Sch"utze, D. A. Hull, and J. O. Pedersen, "A comparison of classifiers and document representations for the routing problem," [6], 1995, Latent semantics indexing and feature selection used as an approach comparison of this approaches will be done. Better performance will be taken.
R. E. Schapire and Y. Singer, "Boostexter: a boostingbased system for text categorization," [7],

2000, Consist of two extensions, specially planned for Multi-class, multi labelled data. In first extension, learned classifier is evaluated to predict a good approximation of sets.

A. Adomavicius, G.and Tuzhilin, "Toward the next generation of recommender systems: A survey of the stateof-the-art and possible extensions," [8], 2005, Recommender system's Over view is explained. Main three approach used in present generation of recommendation system is hybrid, content based and collaborative recommendation. Many restrictions of this system are elucidated. But argue to enlarge the advance system of recommender. So that, this system can be used in wide variety. Extensions embrace sympathetic of users are enhanced, integrating the contextual information in recommendation method, sustain for multi criteria ranking.

B. Sriram, D. Fuhry, E. Demir, H. Ferhatosmanoglu, and M. Demirbas, "Short text classification in twitter to improve information filtering," [9], 2010, In online services like twitter users may grown to be plagued by the rare data. Resolution of this crisis is short text messages classification. To solve this problem, we suggest a small set of domain specific feature is haul out from user profile. This approach successfully classifies the text into generic classes.

V. Bobicev and M. Sokolova, "An effective and robust method for short text classification," [10], 2008, Classification of text encloses complex and specific terminology; need the application of learning method. Partial Matching method is applied which condense the text for confining the text feature. Partial matching develops a language model. The output of partial matching compression provides consistent precision of text classification.

J. Golbeck, "Combining provenance with trust in social networks for semantic web content filtering," [11], 2006, Social network is the common interest grouping web. To make the trust many explanations are required. Two level approaches are stated to combine annotation, trust and provenance. We state an algorithm for concluding trust relationship with provenance information and trust annotation in web social network. Film trust application is introduced which uses trust to movie rating and ordering the review. We consider film trust give the good output.

M. Carullo, E. Binaghi, and I. Gallo, “An online document clustering technique for short web contents," [12], July 2009. Clustering of document is useful in many fields. Two categories of Clustering general purpose and text oriented, these both will be used for clustering of data. Novel heuristic online document clustering is anticipated, which is expert in clustering of text oriented parallel measures. Presentation measure is done in F-measure, and then it will be match up with methods. The result will indicate the power of proposed system. 


\section{CONCLUSION}

In this paper, we propose a system to filter unwanted messages from OSN walls. On-line Social Networks (OSNs) is to give users the ability to control the messages posted on their own private space to avoid any unwanted content to be displayed on the user wall. Up to now OSNs provide little support to this requirement. To fill the gap, we enhance the system by creating an instance randomly notifying a message system that should instead be blocked, or detecting modifications to profile attributes that have been made for the only purpose of defeating the filtering system. Additionally, we plan to investigate the use of online learning paradigms able to include label feedbacks from users. Moreover, the flexibility of the system in terms of filtering options is enhanced through the management of BLs. In particular, future plans contemplate deeper investigation on two interdependent tasks. The first task concerns the extraction and/or selection of contextual features that have been shown to have high discriminative power. The second task involves the learning phase. Since the underlying domain is dynamically changing, the collection of pre-classified data may not be representative in the longer term. The present batch learning strategy, based on the preliminary collection of the entire set of labelled data from experts, allowed an accurate experimental evaluation but needs to be evolved to include new operational requirements. In future work, we plan to address this problem by investigating the use of on-line learning paradigms able to include label feedbacks from users. Additionally, we plan to enhance our system with a more sophisticated approach to decide when a user should be inserted into a BL. The development of a GUI and a set of related tools to make easier BL and FR specification is also a direction we plan to investigate, since usability is a key requirement for such kind of applications. We would like to remark that the system proposed in this paper represents just the core set of functionalities needed to provide a sophisticated tool for OSN message filtering. Moreover, we are aware that a usable GUI could not be enough, representing only the first step. Indeed, the proposed system may suffer of problems similar to those encountered in the specification of OSN privacy settings. As future work, we intend to exploit similar techniques to infer BL rules and FRs. Additionally, we plan to study strategies and techniques limiting the inferences that a user can do on the enforced filtering rules with the aim of bypassing the filtering system, such as for instance randomly notifying a message that should instead be blocked, or detecting modifications to profile attributes that have been made for the only purpose of defeating the filtering system.

\section{FUTURE ENHANCEMENT}

I plan to study strategies and techniques limiting the inferences that a user can do on the enforced filtering rules with the aim of bypassing the filtering system, such as for instance randomly notifying a message that should instead be blocked, or detecting modifications to profile attributes that have been made for the only purpose of defeating the filtering system.

\section{REFERENCES}

[1] Ms. Shruti C. Belsare, Prof. R.R. Keole, "OSN user filtered walls for unwanted messages using content mining", IJCSMC, vol.3, issue 3, march-2014, pg 97-103.

[2] Dipali D. Vidhate, Ajay P. Thakare,”to avoid unwanted messages from osn user wall: content based filtering approach", IJCSMC, VOL.3, Issue.4, april 2014,pg 688-692.

[3] R. J. Mooney and L. Roy, "Content-based book recommending using learning for text categorization," in Proceedings of the Fifth ACM Conference on Digital Libraries New York: ACM Press, 2000, pp. 195-204.

[4] M. Vanetti, E. Binaghi, B. Carminati, M. Carullo, and E. Ferrari, "Content-based filtering in on-line social networks," in Proceedings of ECML/PKDD Workshop on Privacy and Security issues in Data Mining and Machine Learning (2010)

[5] F. Sebastiani, "Machine learning in automated text categorization," ACM Computing Surveys, vol. 34, no. 1, pp. 1-47, 2002.

[6] H. Sch"utze, D. A. Hull, and J. O. Pedersen, "A comparison of classifiers and document representations for the routing problem," in Proceedings of the 18th Annual ACM/SIGIR Conference on Resea. Springer Verlag, 1995, pp. 229-237.

[7] R. E. Schapire and Y. Singer, "Boostexter: a boosting-based system for text categorization," Machine Learning, vol. 39, no. 2/3, pp. 135-168, 2000.

[8] A. Adomavicius, G.and Tuzhilin, "Toward the next generation of recommender systems: A survey of the state-of-the-art and possible extensions," IEEE Transaction on Knowledge and Data Engineering, vol. 17, no. 6, pp. 734-749, 2005.

[9] B. Sriram, D. Fuhry, E. Demir, H. Ferhatosmanoglu, and M. Demirbas, "Short text classification in twitter to improve information filtering," in Proceeding of the 33rd International ACM SIGIR Conference on Research and Development in Information Retrieval, SIGIR 2010, 2010, pp. 841-842.

[10] V. Bobicev and M. Sokolova, "An effective and robust method for short text classification," in AAAI, D. Fox and C. P. Gomes, Eds. AAAI Press, 2008, pp. 1444-1445.

[11] J. Golbeck, "Combining provenance with trust in social networks for semantic web content filtering," in Provenance and Annotation of Data, ser. Lecture Notes in Computer Science, L. Moreau and I. Foster, Eds. Springer Berlin / Heidelberg, 2006, vol. 4145, pp. 101-108.

[12] M. Carullo, E. Binaghi, and I. Gallo, "An online document clustering technique for short web contents," Pattern Recognition Letters, vol. 30, pp. 870-876, July 2009. 